\section{Inter-arm blood pressure differences compare with ambulatory monitoring: a manifestation of the
'white-coat' effect?}

With interest we read the article of Martin et al, ${ }^{1}$ that discussed the use of ambulatory blood pressure monitoring to better understand the clinical significance of interarm differences in blood pressure. We agree it is important to evaluate the diagnostic possibilities for hypertension, especially since it was shown that only half of the time GPs adhere to the guideline of subsequent blood pressure measurements. ${ }^{2}$

The authors state that the whitecoat' effect is probably responsible for the inter-arm differences found in office readings. However, we have some concerns regarding this conclusion. First, a definition of the 'white-coat' effect is not provided by the authors. This is essential for understanding the conclusions drawn in this article. Second, the authors included both patients with a new diagnosis of hypertension as well as patients with already existing hypertension but with failure of treatment, drug intolerance, and/or in need of further investigation of underlying causes. Nevertheless, in the data analysis, these two groups were taken together. We think it is necessary to analyse these groups separately, because Pickering et $a l^{3}$ described a diminishing effect of antihypertensive medication on the 'white-coat' effect. This may also have an effect on the inter-arm differences in blood pressure in the patients with already existing hypertension. This difference even depends on which antihypertensive medication the patient is taking. ${ }^{3}$ However no information on the patients' medication was reported. Furthermore, daytime activities have an effect on ambulatory blood pressure measurements. No information was provided as to whether the patients received instructions on how to spend their day while being monitored.

The authors collected quite some risk factors that are interesting in the context of hypertension. However, the authors did not analyse if any of these factors affected the outcome. If this was the case, it is relevant to correct for these factors in the analysis of the data.

In the results section, information is lacking on whether the first blood pressure was always the highest. If not, this could contradict the authors' explanation of the 'white-coat' effect.

Finally, Figure 2 contains a Bland-Altman plot in which the 'no-difference' line is above zero. Is this a mistake or is there a reason?

Rosa Nieuwenhuize,

Department of General Practice, Erasmus Medical Centre, PO Box 2040, 3000 CA Rotterdam, the Netherlands.

\section{Judith Geuze,}

Department of General Practice, Erasmus Medical Centre, PO Box 2040, 3000 CA Rotterdam, the Netherlands.

\section{Alyt Oppewal,}

Department of General Practice, Erasmus Medical Centre, PO Box 2040, 3000 CA Rotterdam, the Netherlands.

E-mail: a.oppewalderasmusmc.nl

\section{REFERENCES}

1. Martin U, Holder R, Hodgkinson J, McManus R. Inter-arm blood pressure differences compared with ambulatory monitoring: a manifestation of the 'white-coat' effect? Br J Gen Pract 2013; DOI: 10.3399/bjgp13X663055.

2. Heneghan C, Perera R, Mant D, Glasziou P Hypertension guideline recommendations in general practice: awareness, agreement, adoption, and adherence. Br J Gen Pract 2007; 57(545): 948-952.

3. Pickering TG, Gerin W, Schwartz AR. What is the white-coat effect and how should it be measured? Blood Press Monit 2002; 7(6): 293-300.

DOI: 10.3399/bjgp13X667060

\section{Authors' response}

Many thanks for your comments about our recent article. ' We are happy to give a definition of 'white-coat' effect. This phenomenon refers to the transient rise in blood pressure triggered by a visit to the doctor's office, caused by an alerting reaction. The difference between blood pressure measured in the clinic and on ambulatory blood pressure monitoring is usually defined as 'white-coat' effect whereas the clinical situation in which persistently high office blood pressure and normal ambulatory blood pressure coexist regardless of the degree of 'whitecoat' effect is referred to as 'white-coat' hypertension. ${ }^{2,3}$ We agree that it would be interesting to look at the effects of medication on our findings but our study was not set up to do this. Patients on medication referred to our service are complex and usually on multiple medications. It would be impossible to identify differences from individual medication for this reason. We believe such a study would have to be done prospectively. We can confirm that detailed instructions are given to patients when they wear ambulatory monitoring and a patient diary is recorded. We agree that further analysis of the effects of comorbidity on the findings would be interesting to do. We are not able to confirm that the first blood pressure was always the highest as this was not recorded, but data collected since this study suggests that this is often the case. Finally, thank you for pointing out that the 'no-difference' line is above zero on Figure 2; we believe this is a publishing error.

\section{Una Martin}

University of Birmingham, School of Clinical and Experimental Medicine, College of Medical and Dental Sciences, Birmingham, B15 2TT.

E-mail: u.martinabham.ac.uk

Roger Holder,

University of Birmingham, School of Clinical and Experimental Medicine, College of Medical and Dental Sciences, Birmingham.

James Hodgkinson,

University of Birmingham, School of Clinical and Experimental Medicine, College of Medical and Dental Sciences, Birmingham.

\section{Richard McManus,}

University of Oxford, Department of Primary Care Health Sciences, Radcliffe Observatory Quarter, Oxford.

\section{REFERENCES}

1. Martin U, Holder R, Hodgkinson J, McManus R Inter-arm blood pressure differences compared with ambulatory monitoring: a manifestation of the 'white-coat' effect? Br J Gen Pract 2013; DOI: 10.3399/bjgp13X663055.

2. Verdecchia P, Angeli F, Gattobigio R. Clinical 